adenomata stood out more distinctly owing to great diminution in the intervening portions of the goitre. The largest adenoma appeared to have diminished somewhat in size, as its diameter was 34 inches, while that of another was $2 \frac{1}{2}$ inches, or rather more than it was at first. The myxœedematous swelling was notably diminished in all situations. The height had increased by an inch, the temperature had reached the normal level, and the patient was much more lively. About three weeks later the circumference of the neck was 14 inches, a total diminution of 4 inches. The two adenomata previously mentioned had notably diminished in size so that they only measured 2 inches and 1 inch in diameter respectively. The mental condition greatly improved and inany new words and sentences were learned while she was in the hospital. No further increase in height took place. Fig. 4, for which I

FIG. 4.

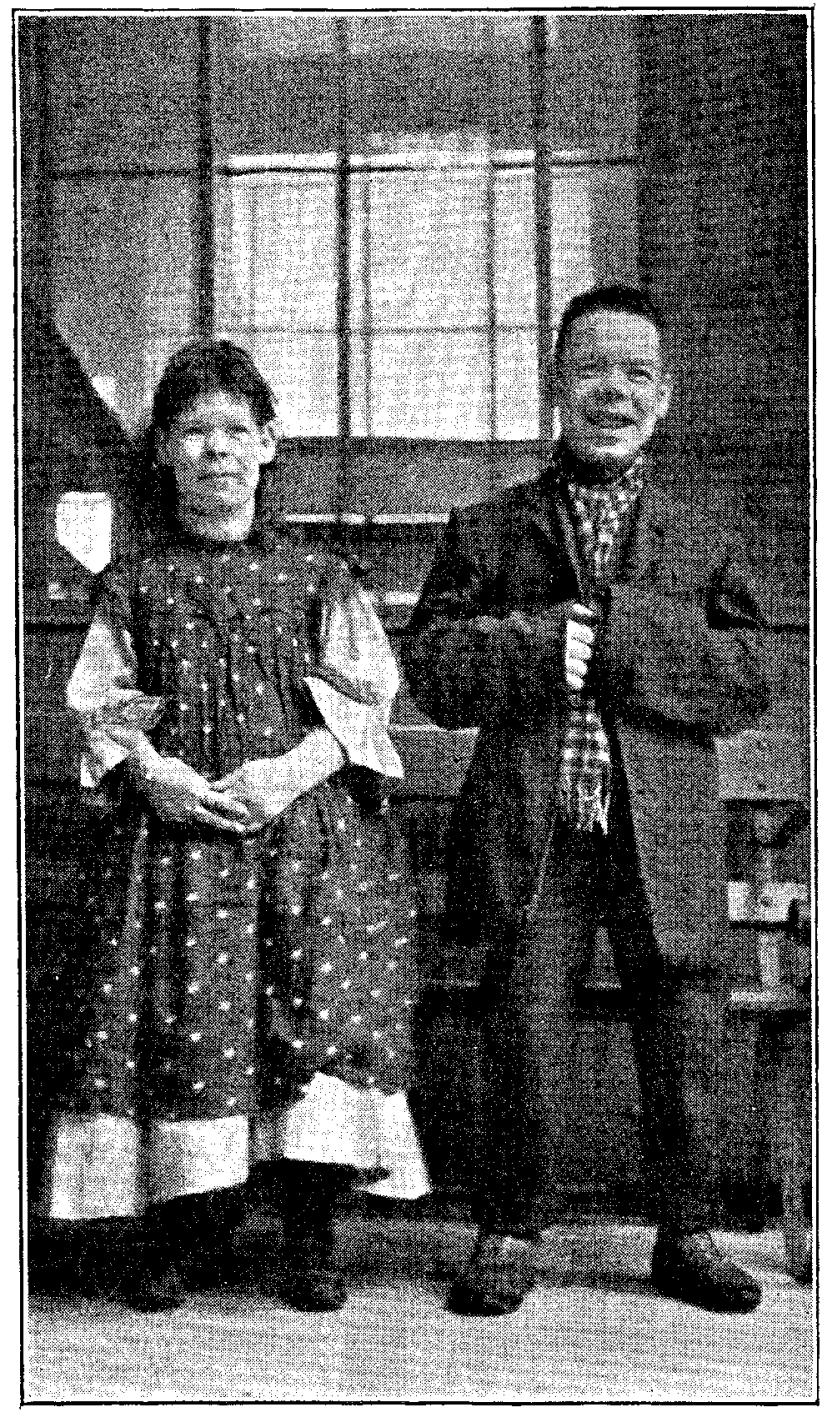

Case $; 2$ and 3 after treatment.

am indebted to Mr. T. B. Eames, my house physician, shows the improvement in appearance in these cases as a result of treatment.

The two adult cases are good examples of the dire results of cretinism when untreated, and thus emphasise the great inportance of the early recognition and treatment of the malady. It is to be hoped that one good result of the systematic medical inspection of school children will be that all cases of cretinism which have escaped recognition before the school age will now be diagnosed and adequately treated, so that in the future we shall not see these advanced cases. The development of the genital organs in the man and regularity of menstruation associated with good mammary development in the woman indicate that they are not examples of the sererest grade of cretinism, in which menstruation is in abeyance and the mammary glands are undeveloped, as in a case previously recorded by me. The improvement in these cases is as much as can be expected in the time when the disease is of such long duration.
The most remarkable feature was the rapid diminution in the size of the goitre, to such an extent that the circum. ference of the neck decreased by four inches. The decrease in size was not only in the parenchymatous part of the goitre, but the adenomata diminished to one-half of their original size. Although we are familiar with the rapid decrease in size in parenchymatous goitre in young adults under the influence of thyroid treatment, I have not before, with one possible exception, seen this take place in adenomata of the thyroid. The result in this case, however, indicates that the treatment is worthy of a more extended trial in cases of adenomatous goitre, as it is possible that in some it may effect a sufficient reduction in size to obviate the necessity for removal by operation.

Manchester.

\section{RABIES, WITH NOTES OF THIRTY CASES.}

BY AD. BAIN, M.D. PARIS,

DIRECTOR OF THE ANTIRABIC INSTYTUTE, CAIRO; AND

WILLIAM J. MALONEY, M.D. EDIN. RESIDENT MEDICAL OFFICER, KASR EL AINY HOSPITAL, CAIRO,

IN Egypt sanitary measures to combat rabies are supplemented by the administration of Pasteur's prophylactio injections to all persons bitten by animals prone to the disease. Native patients while undergoing antirabic treatment are usually inmates of the Government hospital, and afterwards, for one year, they are placed under special observation by their local medical authority, which periodically reports their condition. From October, 1907, to May, 1909,30 cases of human rabies occurred : 17 developed in the course, or at the end of treatment, 10 after leaving, and 3 before entering hospital.

Jackals, dogs, foxes, and their congeners in Egypt form one large hybrid family, the members of which are exposed only to mutual infection, so that the virulence of the rabies organism among them must be fairly uniform, and the relative fatality of their several attacks be dependent mainly upon their respective strength and ferocity. Exceptional potency probably exists, but we found very little evidence of it in experimental inoculation of rabbits. Of our cases, 21 were attributed to bites of dogs, 7 of wolves, ${ }^{1}$ and 2 of animals who attacked in the night and escaped unidentified. of the 27 animals involved, only 16 were traced, and of these none were captured alive. 13 carcasses were received for examination, but all were putrid except 3 . A portion of each brain was introduced intracranially into several rabbits; only two (Experiments 157, 158, and 234, 235) caused rabies, the others septicæmia. Two rabid victims were considered as conclusive evidence of the existence of the disease in each of four untraced animals, but in the remaining 21 animals the diagnosis of rabies rested upon a single reproduction where the possibility of infection previous to the incriminated attack, although highly improbable, could not be positively excluded.

With the exception of two non-fatal man-to-man inoculations, animal bites were the origin of all our rabies infections. Such accidents were usually too complex to have any experi. mental value, but in 11 series of cases common sources of infection were present. In each group of victims of the same animal, wounds of similar severity upon naked sites might be considered to represent approximately equal doses of saliva, although the last wounds probably contained less than the first and those which bled than those which did not; equal amounts of the same saliva represented approximately equal numbers of organisms. The organismal virulence was uniform, and the date of attack and the details of the Pasteur treatment were usually the same for every member of a series. The wounds ranged from skin abrasions to those involving destruction of muscle and bone. Seven degrees of

1 Note by Mr. S. S. Flower, Director of Zoological Gardens, Giza, Fgypt: "Jackal Canis lupaster.-The so-called 'Egyptian wolf' is in termediate in size between the real wolves of northern countries and the jackals of other parts of Africa and Asia. It appears to be numerous throughout Lower and Middle Egypt, and is sald to be destructive to the sugar-cane crop. Specimens in captivity greedily eat dates, grapes, and cherries when they get the opportunity of doing so. This species probably crosses with the local pariah dogs; individual animals may be met with that are difficult to assign to jackal or dog, and are possibly of
mixed parentage." 
severity were distinguished ${ }^{2} ;$ multiple wounds were classified according to the most severe ; multiplicity of similar wounds at the same site could only increase the extent of the infected area, so multiple were considered as extensive wounds with which they were raised to the next degree above an average-sized single wound of corresponding depth ; but as "multiple" in our cases comprised from two to eight, and as the minimum which justified the term "extensive" was ill-defined, the classification afforded only a rough index of the relative severity of the various wounds.

The following brief notes of each series show the influence of various factors upon the result of inoculation:-

First series.-No. 1202, boy, aged 15 years, bitten to the third degree on the face, and third, also, on the hand; died, incubation 41 days; abscesses at injection sites. No. 1203 , man, aged 25 years, bitten on the forehead to the second and on the forearm to the fourth degree; died, incubation 46 days. Each was infected under somewhat similar conditions of dose and site, and had approximately the same incubation; both lived more than 40 days after fairly severe infection, so they must have possessed, at least, average resistance. Although not impossible, it was extremely unlikely that two cases in which resistance could not be enhanced to immunity should simultaneously occur, so the conclusion seems in evitable that the Pasteur treatment here completely failed; the development of abscesses from the injections might account for the five days earlier evolution in No. 1202.

Second series.-No. 1210, boy, aged 13 years; wound of the second degree on his naked foot; survived. No. 1211, brother, aged 12 years; wound of the fifth degree on the face and of second on his naked forearm; died, incubation 22 days. The inoculation in the first was either less than his natural resistance, or from its site and amount powerless to reproduce the disease within the period in which the treatment could not ensure safety. The second obviously received the more massive infection, which alone might adequately explain his rapid death ; at least, there is here no proof of any causal connexion between the site on the face and the shortness of the incubation.

Third series. -No. 1267, boy, aged 10 years, bitten to the fifth degree on his naked finger; died, incubation 33 days. No. 1268 , boy, aged 11 years, bitten through his clothes to the same degree on the forearm; survived. Here, only two factors, the dose and the site, varied; the wounds were of approximately equal severity, but that of the second represented the lesser dose, for it was inflicted by teeth previously cleansed by passing through clothes; the naked hand received a fatal infection, the clothed forearm did not, but there was no evidence of any lethality peculiar to the hand.

Fourth series.-No. 1356, youth, aged 19 years, bitten to the fifth degree on the face; died, incubation 18 days. No. 1357, boy, aged 12 years. bitten through his clothes on the forearm to the second degree; survived. All factors favoured a massive dose in the former and a small dose in the latter. Again, the face was the lethal site, but the extent of the wound was in accord with the fatal infection, and probably also with the shortness of the incubation, so no conclusions could be made regarding the influence of the site.

Fifth serits.-No. 1473, boy, aged 12 years, bitten to the fourth degree on the face; died, incubation 17 days. No. 1474, boy, aged eight years, bitten to the third degree on the naked hand; survived. The difference in dose again obscured the influence of the respective sites, but the face was associated with the graver and fatal wound, and the disease had a short incubation.

Sixth series.-No. 1096, man, aged 23 years, bitten to the fifth degree on the face; died, incubation 23 days. No. 1097, man, aged 30 years, bitten on the leg, through his clothes, to the fifth degree; discharged well, and could not afterwards be traced. No. 1098, girl, aged six years, wound of the fifth degree on the face; died, incubation 105 days. Of the three wounds, two were on the face and proved fatal.

2 Classification of wounds.-1. Single and multiple abrasions of the superficlal layers of the skin; no case occurred in which the wounds belonged solely to this cater trating the skin but cat trating the skin but not underlyng muscles (Case No. 21) 3. Multiple and extensive wounds of the second degree (Cases No. 8, 26, and 28). $19,20$, and 30$)$ and lacerrted wounds of muscles (Cases No. 3, 9, 14, 17, (Cases No. 2,5, Multiple and extensive wounds of the fourth degree (Cases No. 2, 6, 7, 10,13,15, and 16). 6. Wounds involving loss or destruction of muscle, cartilage, or bone (Cases No. 1, 4, 12, 23, and 25). and 29).
The child survived thrice as long as the man, yet in both the disease evolved under identical conditions of dose and site. The child's wound sloughed; the rabies infection may have been uninfluenced or mechanically diminished by the process, but it may also have been reinforced by decreasing the natural resistance to the disease or retarding or preventing the immunisation by the Pasteur treatment; intensive methods would not have affected the result for there was no urgency, and the routine method entirely failed unless the remarkable prolongation of the incubation to 105 days was evidence of an acquired resistance. The man was subjected to a slightly intensive course and died at its conclusion; the treatment may here have hastened death, but it is unlikely that an influence which in the child either prolonged or did not affect the incubation should, when intensified in the man, shorten it. In this series, therefore, the man seemed to be much more susceptible than the child.

Serenth scries.-No. 1186, boy, bitten on the arm, through his clothes, to the second degree; survived. No. 1187, boy, aged 14 years, bitten on the cheek to the third degree; survived. No. 1188, boy, aged 10 years, bitten on the face to the fifth degree; died, incubation 34 days. The features of this series were the survival of the second-a face-biteand the death of the third when presumably on the borders of safety. The dog's teeth were cleansed by the clothes of No. 1186 who was first attacked, so that the others received infections disproportional to their injuries. The second case disproved in his person any special lethality of face inoculations. The same treatment was used for all three and failed in the third, although the incubation was 34 days.

Enghth series.-No. 1259, man, aged 20 years; cauterised wound of the fifth degree on the face; died, incubation 44 days. No. 1260, man, aged 55 years, bitten through his clothes; cauterised wound of the fourth degree on the leg; died, incubation 81 days. No. 1261, woman, aged 60 years, bitten through ber clothes; cauterised wound of the fifth degree on the forearm; fate unknown. Cauterisation by heat was not used till the third day after infection and the result seemed to show it was then useless. The obvious difference in the factors of dosage probably accounted for the difference in the incubation. The Pasteur treatment was not begun till the fourth day, but as the incubation in both was prolonged the failure of the treatment to ensure immunity was absolute and inexplicable.

Ninth series.-No. 1574, man, aged 70 years, bitten to the fifth degree on the wrist and to the same degree on the face ; died, incubation 26 days. No. 1575, man, aged 40 years, bitten to the sixth degree on the hand; survived. No. 1601, boy, aged 12 years, nasal cartilages, both lips, the greater part of both cheeks, and the upper central incisors bitten off ; also six wounds of the fourth degree on the scalp and ear and 14 of the third degree on the forearm. The boy was first attacked, the old man last. The men began Pasteur treatment within 24 hours of the accident; the boy three weeks later. The death of the first, although bitten to a less degree than the second, might indicate a greater lethality for bites on the face than on the hand, but, although from the clinical aspect and from experimental inoculation of rabbits with the patient's brain death was undoubtedly due to rabies, yet the patient suffered from pyonephrosis, which might have lowered his natural resistance and hastened his end. The boy's injuries may have conveyed an exaggerated impression of the dose he received, for a considerable part of the infected tissue of his face was torn off in the attack, but the wounds on his scalp, ears, and arm must have represented an incomparably larger dose than in any other of our fatal cases; the only possible explanation of his survival is that he was naturally immune to rabies.

Tenth series. - No. 1249, boy, aged 15 years, bitten to the seventh degree on the face; died, incubation 19 days. No. 1250 , man, aged 20 years, bitten to the sixth degree on the face; died, incubation 16 days. No. 1251, man, aged 40 years, bitten to the third degree on the naked foot; died from dysentery 50 days after the accident. No. 1252, man, aged 25 years, bitten through his clothes on the knee to the second degree; survived. No. 1253 , man, bitten through his clothes on the thigh to the third degree; survived. The last two were bitten 24 hours after the others. All beyan Pasteur treatment within 48 hours, except No. 1253, who delayed till the third day. The most severely injured were the two who were attacked on the face, and they alone died. The remarkable 
shortness of the incubation might have been due to abnormal organismal virulence or to the site of infection; it seems unlikely that both of those bitten on the face should possess exalted susceptibility and a smaller dose killed the man before the boy. The survival of the other three was probably determined by the trivial extent of their injuries, but the site of infection may also have exerted some influence.

Eleventh series. - No. 1305, man, aged 20 years, bitten to the fifth degree on the hand and to the third on the forearm and shoulder; died, incubation 39 days. No. 1306, boy, aged 15 years, bitten to the fifth degree on the hand and to the second in the face; survived. No. 1307, man, aged 22 years, bitten to the third degree on the hand and to the second on the forearm and leg; survived. No. 1308, man, aged 80 years, bitten to the third degree on the forearm and foot ; reported as dying from old age 26 days after infection. The first received the most massive dose; his antirabic injections were begun within 24 hours of the accident, and failed, although ample time was afforded for the development of immunity. The incubation was relatively long and the inoculation was on a limb, but the organism was probably of feeble virulence, for the second, although wounded to a lesser degree than the first, was still severely injured, and yet he survived in spite of a face infection; the third, who was much less gravely infected, also lived.

The existence of variation in human resistance to rabies was suggested by the sixth and tenth and proved by the ninth series, but the causal factors of this variation were not clearly indicated. Sex was not observed to influence it; social customs and mode of dress shielded Egyptian women from attack, so animal bites among them were infrequent and rabies rare; only four females died and all were children. In the sixth series a girl showed about five times greater resistance than a man, but there was no proof that this was a sexual attribute; and the number of deaths under 11 years was the same in both sexes. As regards age, in the first series the resisvance of 15 years seemed to equal that of 25 ; in the tenth 20 seemed more susceptible than 15 ; in the sixth 23 succumbed nearly five times more quickly than 6 , and in the ninth 12 was immune, 40 survived, and 70 died from rabies complicated by pyonephrosis. At or under the age of 10 years we had eight deaths from rabies, between 10 and 12 years 20 deaths, 20 and 30 years five deaths, 30 and 50 years three deaths, and over 50 years two deaths. The number of deaths at any age was, however, no index of the susceptibility of the period; the incidence of animal bites varied at different ages, for the old, being sedentary, were seldom exposed to attack, whereas the young, being active, gregarious, and unobservant of danger, were especially liable. Towards the extremes of life as the defensive powers diminished the severity of animal bites increased, and as shock, septic processes, and intercurrent diseases frequently complicated bites in the aged, and occasionally in the young, the death-rate from bites was highest in the former and above the average in the latter. But the susceptibility to rabies of different ages was indeterminable, for cases bitten by animals which we proved to be rabid were of insufficient number and of too varying severity to permit comparison.

Our experience seemed to indicate that human resistance, although subject to variations of unknown extent and origin, was usually uniform in childhood and adult life and was unaffected by sex. Concerning old age, we had not enough evidence to justify an opinion.

In every case with the exception of No. 1601 the wound was the most important factor in the result, for upon its severity depended the infecting dose and the number and nature of the absorbing channels. Wounds were separable into two great classes - those on clothed and those on naked sites. The former were comparatively harmless. Thus, in the third series, although two boys received wounds of the same degree, the one bitten through his clothes alone survived. Only one death followed inoculation on a clothed site. Not only did clothes protect the tissues from the biting force, but they also diminished the infection by cleaning the animal's teeth and absorbing its saliva. The latter were always dangerous. It was difficult to prove whether or not the danger varied according to the site. In the fatalities of the second, fifth, and tenth series the face was involved, but with such severity that speculation as to the relative importance of site in them and the survivors was worthless; in the seventh and eleventh series cases survived in spite of wounds on the face, probably because of the slightness of the infections ; while in the ninth, a boy, in whom practically the whole face was bitten off, did not die. of our 30 cases of rabies the face was involved in 26-face alone 21, with forearm 3, and with hand 2 ; the upper limb in 3-upper arm alone 1, upper arm, forearm, and hand 1, and hand alone 1 ; and the lower limb in 1 . These figures are in agreement with the established clinical fact that human rabies usually results from face bites; the face was the most common objective of rabid attacks, and being uncovered and of delicate texture was easily torn. Bites on the face were thus more frequently inoculatious with rabies than those situated elsewhere, and their character was usually such as favoured massive infection and rapid absorption; but we had no proof that any special lethality was peculiar to the face nor that rabies infections of similar severity upon other sites were less liable to end fatally.

In the 17 cases which commenced in hospital the appearance of the first sign traceable only to rabies and followed by further evidence of the disease within 48 hours was taken as marking the end of incubation; by subtracting the average duration of the disease in these from the known interval between infection and death the incubation period of those who died after leaving hospital was calculated. Among the recipients of Pasteur treatment the shortest incubation, 12 days, occurred in a boy, aged 11 years, bitten on the face and scalp to the fifth degree (Case 22); the longest, 105 days, in a girl, aged six years, with a face bite of the fifth degree (Case 6); the oldest patient, 70 years, bitten on the face and wrist to the fifth degree, became rabid within 26 days; the youngest, one and a half years, bitten on the face to the sixth degree, within 22 days. One ( 3.7 per cent.) developed rabies in 12 days, two ( $7 \cdot 4$ per cent.) within 14 days, four (14.8 per cent.) within 17 days, nine $(33 \cdot 3$ per cent.) within 20 days, $15(55 \cdot 5$ per cent.) within 25 days, $21(77 \cdot 7$ per cent.) within 35 days, and 25 (92.6 per cent.) within 50 days. The incubation period was thus inconstant; it oscillated usually between 17 and 35 , occasionally reached 50, but seldom further, and rarely ended before 15; its average duration was 31 days. There was, therefore, in these cases a remarkable and general tendency towards hasty evolutions, although there was no proof of special organismal virulence.

All received injections of unaltered rabies virus, in graduated doses, according to the classic Pasteur method. The routine procedure was to begin with an emulsion in physiological salt solution of a spinal cord of five days' extraction and to continue at intervals of one day with a sequence of cords aged respectively $4,3,5,4,3,2,1,5,4$, $3,2,1,4,3,2,1,4,3,2,1$. The treatment occupied 21 days. Each patient daily received one injection. The injections were begun in 7 cases within 24 hours of the bite and in 20 within 48. In 4 they were delayed till the third day and in 3 till the fourth ; the incubation periods of the former being $17,22,30$, and 32 , and of the latter 19,44 , and 81 days rea spectively. The treatment was interrupted or closed by death in 15 cases. After the end of the treatment a minimum interval of 15 days must elapse before natural resistance can be sufficiently enhanced to ensure safety ; 6 died within this interval; in the remaining 6 , as there was ample time for immunity to develop, the failure of the treatment was com. plete. We could seldom experimentally prove suspected animals to be rabid and we could not ascertain the number of cases of rabies infection which underwent treatment, so the precise measure of our insuccess with the Pasteur treatment was indeterminable. The occurrence of only 6 cases of absolute failure might rightly be construed in favour of the antirabic injections, for with these six exceptions all potential cases of rabies of longer incubation than 36 days were presumably safeguarded. Before the rapid evolution of the disease the treatment was admittedly futile and con. ceivably baneful in 21 cases, in which, however, there was no evidence of the injections hastening the onset of rabies. The remarkable tendency to abbreviation of the incubation period which we observed possibly emphasised the value of the Pasteur treatment, for fulminating cases were beyond its shelter, and the extent to which they figured in our mortality was an index of the success of the prophylaxis in those cases to which it was professedly applicable. We had practically no experience of intensive modes of treatment.

The three cases admitted rabid and untreated by the Pasteur method were remarkable for the range of their 
inoubation. The first, a fellah aged about 40 years, was admitted 18 days after receiving a dog-bite of the fifth degree on the face. According to his own statement and that of his friends, he had then been suffering for seven days from pain in the throat and difficulty in swallowing, and for five days had refused all food and drink; his incubation seemed thus to have been scarcely 12 days (Case 28 ). The second, a boy aged 14 years, had a dog-bite of the fourth degree on the face and developed rabies in 30 days (Case 29). The third, an intelligent artisan aged about 45 years, corroborated by his three adult sons, positively affirmed that his only exposure to infection occurred 18 months before admission, when he was bitten on the left upper arm by a dog. The wound was inflicted through his clothes and was cauterised within an hour of the accident. The infection might not have dated from this attack, but he had only one scar, an old white, irregularly oval cicatrix, with two eccentric depressions, situated near the left deltoid insertion.

Early diagnosis of rabies was frankly tentative. Pyrexia was the most frequent initial sign, but it was inconstant and occasionally rose from obscure causes unrelated to rabies. Mental unrest, a common early sign, was unreliable, for even among non-rabid patients of average nervous stability restlessness and anxiety sometimes accompanied antirabic treatment. Pain in the throat with difficulty in swallowing was the first indication in three cases; cardiac irregularity in one; and in another difficulty in walking. Usually fever with emotional disturbances were quickly followed by the classical laryngeal symptoms and the disease rapidly evolved so as to place the diagnosis beyond doubt; but simple throat infections with fever, pain and difficulty in swallowing, and acute anxiety were sometimes quite indistinguishable from incipient rabies. In one case a temperature of unknown origin and great perturbation were followed by marked hesitancy in drinking (No. 1601) and in another by definite laryngeal spasm (No. 1471); both were suspected of rabies, their Pasteur treatment was continued unaltered, they showed no further signs, and completely recovered. In two suspicious cases bitten by a wolf, whose third victim had died rabid, we thought to derive diagnostic aid from the cerebro-spinal fluid. A lumbar puncture was made; the fluid in both was at very high pressure, but it did not reproduce the disease when exhibited intracranially to rabbits. Both patients are still alive. The signs were so protean and the course so anomalous that diagnosis was sometimes uncertain, even after post-mortem examination, without the proof of experimental inoculation. Case 3 developed fever, headache, and mental changes 21 days after infection; he was at first thought to be rabid but no further symptoms arose and his temperature fell; an irregular pyrexia with slight splenic enlargement was the next phase, and the blood was fruitlessly examined for typhoid, paratyphoid, and Malta fevers; pyæmia was then suspected, and on the thirty-eighth day albuminuria and broncho-pneumonia appeared and strengthened this diagnosis; a generalised pyæmic state was found post mortem, but his brain substance produced rabies in rabbits. This may have been an abortive case of rabies which succumbed to pyæmia, or an example of the effect of the super-position of pyæmia upon rabies, but in Case 24 the evolution of the latter disease was uninfluenced by the presence of profound sepsis. The brain substance of persons who are in the incubation stage of rabies or who have completed a course of Pasteur treatment may induce rabies in rabbits; several experiments with brain substance to decide these questions were inconclusive, and the cerebro-spinal fluid of six cases which had undergone the routine series of antirabic injections proved innocuous when introduced subdurally into rabbits. In our cases we did not use histological methods but relied upon the clinical aspect of the disease, the necropsy, and experimental inoculations with the suspected brain to establish the diagnosis of rabies.

In 14 cases the wounds were completely healed and the scars showed no evidence of special irritability when rabies ensued; healing was partial at the beginning of the disease in six, and in three of these it so progressed as to become perfect before death. Wounds in the rabid seemed not to differ from those in the non-rabid; they underwent no change peculiar to the disease, and healing appeared neither to be hastened nor delayed.

Faucal congestion, partial or general, was present in every case : in three, all children, it was trivial ; in seven, slight; and in 10 pronounced. Of the last, three were cases in which much distress arose from accumulation in the upper air passages of viscid mucus which the patient strove to expectorate by a short frequent rasping cough; in these the congestion increased as the disease advanced, and the lower part of the posterior pillars seemed to be most affected. In only five cases was the state of the throat previous to the onset of rabies observed, and in four of these no change occurred. Of 100 consecutive patients, of whom 78 were male and 22 female and 45 were children, who presented themselves here for antirabic treatment during the months of January, February, and March, 1909, only 17 were free from faucal congestion, and of these 13 were children. 83 showed acute and chronic inflammatory processes of varying intensity and extent affecting the fauces, pharynx, and larynx. As faucal congestion is thus without constant characters in the rabid and in no definite causal relationship to the disease, and as it is so frequently present in the nonrabid, it is practically useless as a sign of rabies.

Salivation was not an early sign; it usually appeared on the eve of the second or in the course of the third day and the secretion increased as the disease progressed; the viscidity varied partly but not entirely as the amount of the secretion; the viscid forms were sometimes accompanied by a troublesome cough and faucal, pharyngeal, and laryngeal congestion; copious salivation usually involved frequent expectoration with frothing at the lips in the rabid stage and dribbling in the paralytic. In two cases in which salivation was slight when the paralytic stage developed and the tongue lay motionless in the dropped jaw the palate was seen to be studded by little pearly beads of secretion. In three cases no increase of saliva was noted, in seven the increase was slight but obvious, and in ten it was marked. In two cases the parotids, in three the submaxillary salivary, and in two the submaxillary lymphatic glands were enlarged; in two the tonsils were hypertrophied. Lacrymation was only thrice noted.

In the 17 cases in which we observed the commencement of the disease an initial rise of temperature occurred in 15 ; of these, two were septic and a third had a slight irregular fever, so only in 12 could no cause except the onset of rabies be found for the pyrexia; but in 14 the onset and rise appeared simultaneously and in the fifteenth the onset synchronised with an access of fever. In two the initial rise was absent; in 7 it varied from $0.4 \circ$ to $0.9 \circ$, and in 6 from $1.0 \circ$ to $1.5^{\circ}$; its maximum was $1.5^{\circ}$ and its average $0.9 \circ$. The course of the temperature varied with the duration of the disease; in cases lasting only one or two days the fever rose till death; in 13 cases it reached its height on the second day, in two on the fourth, in one on the third, and in one on the first. The maximum rise in three cases one septic-was less than $1^{\circ}$; in three between $1.0^{\circ}$ and $1.5^{\circ}$; in 13 -one septicbetween $2.0^{\circ}$ and $2.9^{\circ}$; and in one $4.3^{\circ}$, above normal. In nine an agonal rise of varying extent occurred and in nine a fall; in two, no temperature change immediately preceded death. As a rule the more acute the case the higher was the temperature; if the rabid stage predominated the case was usually but not invariably febrile. In two out of the three cases in which the period of excitation was only slightly marked pyrexia accompanied the paralytic stage, which, however, in its usual sequence, following a more or less exhausting rabid stage, was characterised by a temperature with a subnormal tendency which doubtless had its origin partly in starvation. A morning fall and evening rise were customary, but not invariable, and the extent of the daily temperature excursion had no characteristic uniformity. In prolonged cases the fever usually reached its height on the second day and then progressively sank to lower levels till temperatures of $36^{\circ}$ or even $35^{\circ}$ were attained and the disease continued at such low levels till the end. (Of the above temperatures the initial were mouth, the others rectal ; all were Centigrade.)

of 20 cases only 16 showed laryngo-pharyngeal symptoms. of these, one complained of pain only in swallowing, which was accomplished with apparent ease; three showed a certain degree of hesitancy and deliberation in drinking which was preceded by several gasping inspirations ; six performed the act with a slight but definite jerk, and six showed marked spasm. The severity of the spasm varied : in slight cases the respiratory and pharyngeal muscles alone were involved; in graver cases a more or less general clonic convulsion occurred, and in one case a generalised tonic spasm of short duration with a certain degree of opisthotonos resulted; 
in two cases incontinence of urine was observed. The severity of the spasm was somewhat dependent upon the inducing stimulus: in minor cases an attempt at drinking was the minimal and only stimulus and produced the maximal effect; in graver cases the sight, sound, or suggestion of fluid sufficed to produce a spasm, but the maximal effect was usually not achieved unless an effort to drink was made. The lesser degree of spasm still permitted drinking after a more or less painful ordeal, in which the patient usually averted his eyes, shakily grasped the drinking vessel, jerkily raised it to his lips, and then pansed tremulously while he made several attempts to induce the necessary muscular coördination by swallowing his saliva. In almost every case irregular, gasping inspirations immediately preceded the actual attempt. In the more severe degrees an elaborate ritual was observed before the fluid was negotiated: the patient punctiliously arranged his surroundings, carefully adjusted his clothing, and dismissed any unwelcome spectators; no effort was made to swallow saliva, but the mouth and throat were repeatedly cleared by expectoration; then eager, earnest prayers were jerked forth, under cover of which abortive attempts were made to take the muscles of swallowing by surprise ; finally some fluid was actually introduced into the mouth; a violent spasm at once resulted and the fluid was forcibly expelled.

The intensity of the spasm occasionally made the patient scream with agony. If the pain was marked the patient needed to be persuaded to drink, but if it was slight he sought the test and strove to repeat it in the hope that each mouthful would prove his difficulty to have disappeared. Solids were always more readily negotiated than fluids and could usually be swallowed in small quantities even in the presence of violent spasm; neither the sight of solid food nor the suggestion of eating induced the spasm in any of our cases.

The voice was usually clear and shrill, but in those who had the typical cough hoarseness was usual, and one patient was practically aphonic. Speech was punctuated by gasps and rapid or tempestuous according to the degree of excitement, but in several cases towards the height of the rabid period speaking seemed painful and difficult and responses were slow and of few words.

Laryngo-pharyngeal symptoms were the first indication of the disease in three cases; they appeared within 24 hours in 11 , within 48 in 13 , and were entirely absent in four; in the three who were admitted rabid, spasm was marked but its precise date of development was uncertain. Pain, hesitancy, gulp, and spasm were noted successively to develop in two instances, so each may mark an inconstant stage in a process which varies in its completeness. Usually these symptoms progressively advanced towards a maximal severity, but sometimes they waned and waxed from hour to hour and even so completely disappeared as to give rise to false hopes of a mistaken diagnosis. With the onset of the quiescent period the spasm abated, paralysis ensued, and the power of swallowing was more or less abolished. These symptoms were a very valuable aid to diagnosis, but in the early stages they were difficult to distinguish from the symptoms of slight septic throat affections; in two cases they were present and no other sign of rabies followed, and in four, in which the brain substance induced rabies in rabbits, they were entirely absent.

Psychological changes were of constant and early occurrence, but as all our patients were Egyptians, delicate initial changes were difficult to detect and nnobtrusive mental symptoms were not observed unless some obvious danger signal, such as fever, had first focussed attention. The earliest complaints were of great weariness, headache, stabbing pain or sense of constriction in the chest, and pain in the throat. Usually suppressed excitement and fear first developed and were succeeded or obscured by a state of extreme dejection and misery ; surliness, silence, and weeping were not common; dread of change and of their fellow patients, disinclination for food and fear of drink, sleeplessness, and delusions culminated not infrequently in furious outbursts. These bouts were sometimes the climax of a period of progressively increasing irritability in which the patient was often reduced to an agony of vigilance by the idea of his impending murder by stabbing; attendants, parents, friends, all were suspected. One patient sought safety by locking and defending the door of his ward, another by attempting to jump out of the window, and two others by trying to escape from hospital. Several were terrorised by pursuit by imaginary men and animals, and were liable to maniacal attacks on the slightest additional stimulus. Rarely such outbursts appeared with startling suddenness in the midst of a period of apparent quiescence. In children they were expressed by fits of screaming with convulsions, or intense restlessness. These attacks often ceased as suddenly as they began, and interludes in which the patient was calm, free from fear, of assured wellbeing, and of hopefulness verging almost on gaiety occurred in a bewildering fashion. Consciousness in children seemed early to disappear, but in adults it was usually maintained, sometimes with appalling clearness, to the end; hence some cases with a marked tendency to violence were readily soothable. Two in whom reason was lost coursed round their room on all fours emitting short, shrill frequent cries and seemed indifferent to or resentful of attempted interference : in these the virus may have specially affected the cerebellum; four were so violent that mechanical restraint was necessary. As the disease advanced the rabid outbursts became milder and the intervals of apathy and exhaustion more prolonged till they gradually merged into death.

Occasional vomiting was thrice noted. Irregularity in the force and rhythm of the heart was a late symptom in seven cases, and in two was present from the onset. The heart-rate varied with the age of the patient and the stage of the disease; usually it was at first accelerated, but later there was a tendency to slowing; even in markedly febrile cases it seldom rose above 90 per minute, and a rate of from 58 to 65 was not uncommon in mildly febrile cases in adults. Respiration at first quickened : in adults a rate of from 28 to 36 was frequently observed, and in children 40,50 , and in one case 56 per minute. It soon became irregular and interrupted by frequent short sighs; later, it sometimes became a confusion of deep and shallow inspirations with erratic and sometimes prolonged apnceic pauses; sometimes it was deep and slow with movements of the alæ nasi and indrawing of the intercostal spaces. In a child of five years, in whom this last type persisted throughout, the rate was from 16 to 19 per minute. In four children bronchitis complicated rabies.

In spite of the prevailing tendency to melancholia patients were usually voluble in pious expressions of thankfulness for their anticipated recovery. Frequently the more obvious the advance of the disease the more insistent were the protestations of well-being and gratitude. Speech was irregularly punctuated with sudden halts; expression often imperfect, concentration lacking, and word memory faulty; hence some degree of incoherence was frequently present.

There were no characteristic eye signs, but strabismus was four times observed, ptosis twice, and nystagmus and photophobia each once. The fundus was examined in four cases and no changes were found. Exaggerated deep reflexes, clonus, head retraction, and other evidences of spasticity occurred with hyperæsthesia which involved varying areas of the trunk and limbs-especially the lower; later complete flaccidity and paralysis with anæsthesia developed. All degrees of initial irritability and subsequent depression were observed, but occasionally there was neither definite motor nor sensory disturbance. Incontinence of urine was usually present throughout in children; in adults it occurred only at the height of a convulsion or in the paralytic stage ; constipation was usual. Convulsions were once localised to, and twice accentuated upon, the side of the lesion, but in other cases both sides seemed equally affected.

Rarely, as in Case 18, the pre-rabid stage predominated, and after a varying period of gentle melancholia with rapid emaciation the patient died. Sometimes the preliminary irritability suddenly changed to acute mania and death quickly supervened from cardiac failure without the development of any paralytic stage--e.g., Case 12, a man, aged 20 years, was bitten to the sixth degree on the face and had an incubation period of 16 days. For about 14 hours he was anxious, restless, and somewhat tremulous; his speech was rapid, words ran in unequal gasps, but he was very insistent that he was not ill $\mathrm{He}$ had a slight laryngeal spasm; suddenly he became wildly excited and rushed shrieking through the hospital; he was captured, but in the process he bit an attendant severely in the loin. The furious outburst lasted one hour. He became suddenly quiet and on examination was found to be dead. 
In two cases there was practically neither preliminary nor paralytic stage ; e.g., Case 17, a boy, aged five years, was bitten on the face to the fourth degree, and rabies began on the twenty-third day. Two hours after he had been noticed to be "queer and restless" his temperature rose to $38.5^{\circ}$ and continued to rise till death, when it reached $41 \cdot 3^{\circ}$. For eight hours he was quite maniacal, throwing himself violently against the padding of his cot or crouching in a corner screaming with terror and trying to beat off a swarm th of imaginary dogs. He was quite unsoothable. He took a bromide and chloral draught apparently without difficulty or spasm about five hours before death, which occurred with startling abruptness 11 hours after the first symptom. His brain gave rabies to rabbits. In others the paralytic symptoms predominated; e g., Case 6 , a girl, aged six years, was bitten to the sixth degree on the left side of the face and had an incubation of 21 days. This patient had no initial fever but the temperature rose to $39^{\circ}$ at the end of the first day and remained at a high level for 36 hours, when death occurred; she had no laryngeal spasm and mentally at first she seemed quite normal except for some natural anxiety. She suddenly found difficulty in walking. There was nothing distinctive in her gait, but she was too weak to stand and walked clumsily when supported. The knee-jerks were absent, the plantar and abdominal reactions were very feeble, and there was practically no response to heat or pain below the knees. The pulse became irregular, the patient grew gradually more difficult to rouse, and after 18 hours first the left arm and then the left side of the face began to twitch. The arm movements ceased in a few hours but the facial contortions continued and spread to involve the right side. There were no pupillary nor fundal changes but there were first lateral nystagmus and later internal convergence. All sensation and reflexes, except the conjunctival, disappeared. The head was slightly retracted and frequently rotated to the left side. An intermittent muttering of varying loudness was maintained at intervals. Slight salivation and increase of tears were noted. About 10 cubic centimetres of crebro-spinal fluid were drawn off and the convulsions ceased; the pressure of the fluid as measured by a normal saline manometer was 9.2 cubic centimetres; the fluid was clear, contained very few cells, and did not give rabies to rabbits. The pulse-rate increased till it reached 186 per minute; there was no diaphragmatic action; the respirations were regular and shallow, but never exceeded 32 per minute; bronchitis was widespread. About two hours before death there appeared a fish-like gasp of the dropped lower jaw and extension of the head with each inspiration. It was worthy of note that although the lesion was on the face the disease first manifested itself in the legs, then in the arms, and lastly in the face; also, that the convulsive movements were confined almost wholly to the side of the lesion.

Another paralytic type was exemplified in Case 23. A healthy baby girl, aged one and a balf years, was bitten to the sixth degree on the right side of the mouth and had an incubation of 22 days. The infant was sleeping and feeding well. The mother suddenly noticed that "the eyes were staring " and the child "seemed queer." Two hours later it became very restless and tossed about incessantly in its cot. Sudden piercing screams soon followed and then slight but rapidly increasing and almost continuous clutching and twitching movements of the fingers and arms appeared with frequent general convulsions. The power of swallowing was either much diminished or altogether absent. There seemed to be a certain degree of hyperæsthesia, for the slightest touch induced a fit of screaming. The knee-jerks conld not be elicited, and although the legs were briskly drawn away the toe movements on stroking the plantar surface of the feet were slight and indeterminate. Squint and right ptosis were present but no pupillary nor fundal changes. After about nine hours the child quietened and remained collapsed and exhausted all next day. On the third day it seemed somewhat better. Fluid introduced into the mouth mostly dribbled out again but a little may have been swallowed. There were then no deep nor superficial reflexes nor response to pain; winking had disappeared, but the conjunctival reflex was still slightly present. The heart was quite regular; there was slight salivation with dribbling; there was no ferer; the temperature throughout had a markedly sub. normal tendency and progressively sank till on the seventh day when death occurred it was only $35.4^{\circ}$. The post- mortem examination showed no gross lesion, but experimental inoculation of rabbits proved death to have been due to rabies.

Finally, there was a type which somewhat simulated tuberculous meningitis-e.g., Case 19. A boy, aged nine years, was bitten by a dog on the face to the fourth degree and had an incubation of 19 days. The initial temperature was $37.8^{\circ}$; the maximum occurred on the fifth day, $39.4^{\circ}$; then it slowly fell till on the ninth day it was $36 \cdot 80$. Death occurred on the tenth and there was an agonic rise to $38.2^{\circ}$. Pain in the throat, especially on swallowing, was present from the beginning, but the tonsils were enlarged and inflamed and the glands at the angle of the jaw were palpable. On the second day a slight spasm in drinking was noted but it did not reappear. The boy was very fretful and tock neither food nor fluid well. A blood examination on the third day showed a slight polymorphonuclear leucocytosis. The urine was normal. On the fourth day there were a few râles at both bases, the spleen was not enlarged, and the Widal reaction was absent. On the seventh day he was very restless and frequently uttered a short scream; his pulse and respiration were markedly irregular; the abdomen was scaphoid and head retracted. Photophobia and squint were present but no fundal changes. The kneejerks could not be elicited ; the plantar responses were feeble and mainly flexor. Sensation was markedly diminished and there was slight salivation. Lumbar puncture was performed and about 15 cubic centimetres of fluid were drawn off. The cerebro-spinal fluid was at high pressure and faintly milky; it did not cause rabies when injected intradurally. The patient quietened but gradually sank. He became perfectly flaccid and apparently paralysed and insensitive about 36 hours before death. Except for cerebral congestion and œdema the post-mortem examination was negative, but the brain substance gave rabies to rabbits.

The only noteworthy point in the post-mortem examinations was the presence in all but one of marked increase in the cerebro-spinal fluid; the relief of the intracranial tension by lumbar puncture we found in three cases to be of some value in quietening very irritable patients; our six series of experiments with the fluid as a means of ante-mortem diagnosis produced negative results. From the few cases we have cited it is obviously extremely diffoult to assert that any case is suffering from incipient rabies, so alleged cures may be discounted. Treatment after the disease was definitely declared proved invariably futile, but to nasal and rectal feeding perhaps the great prolongation in Cases 19 and 23 was partly due.

We are indebted to the Director-General of the Public Health Department of Egypt for permission to publish this paper.

\section{THE ETIOLOGY OF THAT FORM OF DIS- LOCATION OF THE HIP-JOINT WHICH IS GENERALLY REGARDED AS CONGENITAL.}

BY RALPH THOMPSON, CH.M. VICT., F.R.C.S. ENG.,

SENIOR DEMONSTRATOR OF ANATOMY, GUY'S HOSPITAL MEDICAL SGHOOL; ASSISTANI' SURGEON, YICTORIA HOSPI'AL FOR GHILDREN.

THE object of this paper is to suggest that in some cases that form of dislocation of the hip-joint, which occurs in young infants and children without any apparent violence, and which is usually regarded as congenital, may be in reality due to pressure exerted upon the hip-joint during the adoption of the erect attitude of such a nature as to produce displacement of the head of the normal femur from a nearly normal acetabulum. The large preponderance of cases which occur in the female sex needs an explanation which the usual view of the condition entirely fails to supply. Congenital deformities, especially those due to intrauterine pressure, such as talipes (unassociated with spina bifida) or deformities occurring at birth, are more likely to occur in male children, owing to the larger size of male as compared with femaie children. Other congenital deformities, such as cleft palate, are more common in surviving males than in females.

Professor A. Keith has suggested that the condition is due 